\title{
On Neutrosophic Quadruple Groups
}

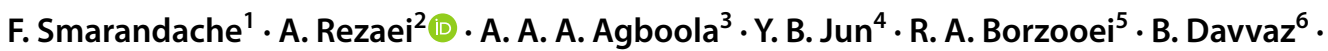 \\ Arsham Borumand Saeid ${ }^{7} \cdot$ M. Akram ${ }^{8} \cdot$ M. Hamidi $^{2} \cdot$ S. Mirvakili ${ }^{2}$
}

Received: 21 January 2021 / Accepted: 2 November 2021

(c) The Author(s) 2021

\begin{abstract}
As generalizations and alternatives of classical algebraic structures there have been introduced in 2019 the NeutroAlgebraic structures (or NeutroAlgebras) and AntiAlgebraic structures (or AntiAlgebras). Unlike the classical algebraic structures, where all operations are well defined and all axioms are totally true, in NeutroAlgebras and AntiAlgebras, the operations may be partially well defined and the axioms partially true or, respectively, totally outer-defined and the axioms totally false. These NeutroAlgebras and AntiAlgebras form a new field of research, which is inspired from our real world. In this paper, we study neutrosophic quadruple algebraic structures and NeutroQuadrupleAlgebraicStructures. NeutroQuadrupleGroup is studied in particular and several examples are provided. It is shown that $(N Q(\mathbb{Z}), \div)$ is a NeutroQuadrupleGroup. Substructures of NeutroQuadrupleGroups are also presented with examples.
\end{abstract}

Keywords Neutrosophic quadruple number $\cdot$ NeutroAlgebra $\cdot$ NeutroQuadrupleGroup $\cdot$ NeutroQuadrupleSubgroup

\section{Introduction}

It was started from Paradoxism, then to Neutrosophy, and afterwards to Neutrosophic Set and Neutrosophic Algebraic Structures. Paradoxism [21] is an international movement in science and culture, founded by Smarandache in $1980 \mathrm{~s}$, based on excessive use of antitheses, oxymoron, contradictions, and paradoxes. During the 3 decades (1980-2020), hundreds of authors from tens of countries around the globe contributed papers to 15 international paradoxist anthologies. In 1995, Smarandache extended the paradoxism (based on opposites) to a new branch of philosophy called neutrosophy (based on opposites and their neutrals) that gave birth to many scientific branches, such as

\author{
A. Rezaei \\ rezaei@pnu.ac.ir \\ F. Smarandache \\ smarand@unm.edu \\ A. A. A. Agboola \\ agboolaaaa@funaab.edu.ng \\ Y. B. Jun \\ skywine@gmail.com \\ R. A. Borzooei \\ borzooei@sbu.ac.ir \\ B. Davvaz \\ davvaz@yazd.ac.ir \\ Arsham Borumand Saeid \\ arsham@uk.ac.ir \\ M. Akram \\ m.akram@pucit.edu.pk \\ M. Hamidi \\ m.hamidi@pnu.ac.ir
}

S. Mirvakili

saeed_mirvakili@pnu.ac.ir

1 Department of Mathematics and Science, University of New Mexico, Gallup, NM 87301, USA

2 Department of Mathematics, Payame Noor University, P.O. Box. 19395-3697 Tehran, Iran

3 Department of Mathematics, College of Physical Sciences, Director ICTREC, Federal University of Agriculture, PMB 2240 Abeokuta, Ogun State, Nigeria

4 Department of Mathematics Education, Gyeongsang National University, Jinju 52828, South Korea

5 Department of Mathematics, Shahid Beheshti University, Tehran, Iran

6 Department of Mathematics, Yazd University, Yazd, Iran

7 Department of Pure Mathematics, Shahid Bahonar University of Kerman, Kerman, Iran

8 University of the Punjab, New Campus, Lahore, Pakistan 
neutrosophic logic, neutrosophic set, neutrosophic probability and statistics, neutrosophic algebraic structures, and so on with multiple applications in engineering, computer science, administrative work, medical research etc. Neutrosophy is an extension of Yin-Yang Ancient Chinese Philosophy and of course of Dialectics. From Classical Algebraic Structures to NeutroAlgebraic Structures and AntiAlgebraicStructures. In 2019 and 2020, Smarandache [16-18] generalized the classical Algebraic Structures to NeutroAlgebraicStructures (or NeutroAlgebras) whose operations and axioms are partially true, partially indeterminate, and partially false as extensions of Partial Algebra, and to AntiAlgebraic Structures (or AntiAlgebra) whose operations and axioms are totally false. By considering a space and an operation defined on, in general, it does not mean that the operation is well defined for all elements of the space. We have three cases, as in neutrosophy: either the operation is well defined (as in classical algebraic structures), or partially defined and partially undefined, or partially outer-defined. Similarly, in general by defining an axiom on a given space under some given operations it does not mean that the axion is true for all elements of the space. Again we gave three cases as in neutrosophy: the axiom is true for all elements (as in classical algebraic structures), or the axiom is partially true and partially false, or the axiom is false for all elements. Motivation is the fact that in mathematics, in general, by defining an operation on a given set it does not mean that the operation is automatically well defined, but many times it is only partially well defined. Similarly, by defining an axiom on a given set, in general it does not mean that the axiom is true for all elements, but only partially true (i.e. true for some elements and maybe false for other elements). In the present paper, we study neutrosophic quadruple algebraic structures and NeutroQuadrupleAlgebraicStructures. NeutroQuadrupleGroup is studied in particular and several examples are provided. It is shown that $(N Q(\mathbb{Z}), \div)$ is a NeutroQuadrupleGroup. Substructures of NeutroQuadrupleGroups are also presented with examples.

\subsection{Operation, NeutroOperation, AntiOperation}

When we define an operation on a given set, it does not automatically mean that the operation is well defined. There are three possibilities:

- The operation is well-defined (or inner-defined) for all set's elements (as in classical algebraic structures this is classical Operation).

- The operation if well-defined for some elements, indeterminate for other elements, and outer-defined for others elements (this is NeutroOperation).

- The operation is outer-defined for all set's elements (this is AntiOperation).

\subsection{Axiom, NeutroAxiom, AntiAxiom}

Similarly for an axiom, defined on a given set, endowed with some operation(s). When we define an axiom on a given set, it does not automatically mean that the axiom is true for all set's elements. We have three possibilities again:

- The axiom is true for all set's elements (totally true) (as in classical algebraic structures; this is a classical Axiom).

- The axiom if true for some elements, indeterminate for other elements, and false for other elements (this is NeutroAxiom).

- The axiom is false for all set's elements (this is AntiAxiom).

\subsection{Algebra, NeutroAlgebra, AntiAlgebra}

- An algebraic structure whose all operations are welldefined and all axioms are totally true is called Classical Algebraic Structure (or Algebra).

- An algebraic structure that has at least one NeutroOperation or one NeutroAxiom (and no AntiOperation and no AntiAxiom) is called NeutroAlgebraic Structure (or NeutroAlgebra).

- An algebraic structure that has at least one AntiOperation or Anti Axiom is called AntiAlgebraic Structure (or AntiAlgebra).

Therefore, a neutrosophic triplet structure is formed (see [1-8]):

$<$ Algebra, NeutroAlgebra, AntiAlgebra $>$.

"Algebra" can be: groupoid, semigroup, monoid, group, commutative group, ring, field, vector space, BCK-Algebra, BCI-Algebra, K-algebra, BE-algebra, $H_{v}$-rings, etc. (see [9-15] and [20]).

The sets of natural/integer/rational/real/complex numbers are, respectively, denoted by

$\mathbb{N} \subseteq \mathbb{Z} \subseteq \mathbb{Q} \subseteq \mathbb{R} \subseteq \mathbb{C}$

The Neutrosophic Quadruple Numbers and the Absorbance Law were introduced by Smarandache [19]; they have the general form:

$N=a+b T+c I+d F$, where $a, b, c, d$ may be numbers of any type (natural, integer, rational, irrational, real, complex, etc.), where " $a$ " is the known part of the neutrosophic quadruple number $N$, while " $b T+c I+d F$ " is the unknown part of the neutrosophic quadruple number $N$; then the unknown part is split into three subparts: degree of confidence $(T)$, degree of indeterminacy of confidence-nonconfidence $(I)$, 
and degree of nonconfidence $(F) . N$ is a four-dimensional vector that can also be written as: $N=(a, b, c, d)$.

There are transcendental, irrational, etc. numbers that are not well known, they are only partially known and partially unknown, they may have infinitely many decimals. Not even the most modern supercomputers can compute more than a few thousands decimals, but the infinitely many left decimals still remain unknown. Therefore, such numbers are very little known (because only a finite number of decimals are known), and infinitely unknown (because an infinite number of decimals are unknown). Take for example: $\sqrt{2}=1.4142 \ldots$.

\section{Arithmetic Operations on the Neutrosophic Set of Quadruple Numbers}

Definition 1 A neutrosophic set of quadruple numbers denoted by $N Q(X)$ is a set defined by

$N Q(X)=\{(a, b T, c I, d F): a, b, c, d \in \mathbb{R}$ or $\mathbb{C}\}$,

where $T, I, F$ have their usual neutrosophic logic meanings.

Definition 2 A neutrosophic quadruple number is a number of the form $(a, b T, c I, d F) \in N Q(X)$. For any neutrosophic quadruple number $(a, b T, c I, d F)$ representing any entity which may be a number, an idea, an object, etc., $a$ is called the known part and $(b T, c I, d F)$ is called the unknown part. Two neutrosophic quadruple numbers $x=(a, b T, c I, d F)$ and $y=(e, f T, g I, h F)$ are said to be equal written $x=y$ if and only if $a=e, b=f, c=g, d=h$.

Example $1 N Q(\mathbb{N}), N Q(\mathbb{Z}), N Q(\mathbb{Q}), N Q(\mathbb{Z})$ and $N Q(\mathbb{C})$ are neutrosophic sets of quadruple natural, integers, rationals, real and complex numbers respectively.

Example 2 The following

$x=2-3 T+4 I-5 F \in N Q(\mathbb{Z})$,

$y=\sqrt{2}-\frac{3}{4} T-11 I-\frac{5}{6} F \in N Q(\mathbb{R})$,

$z=(3+2 i)-(-4+3 i) T+(4 i) I-\left(\frac{1}{5}-\frac{1}{6} i\right) F \in N Q(\mathbb{C})$

are examples of neutrosophic quadruple of integers, real and complex numbers, respectively.

Definition 3 L e t $a=\left(a_{1}, a_{2} T, a_{3} I, a_{4} F\right)$, $b=\left(b_{1}, b_{2} T, b_{3} I, b_{4} F\right) \in N Q(X)$. We define the following:

$a+b=\left(a_{1}+b_{1},\left(a_{2}+b_{2}\right) T,\left(a_{3}+b_{3}\right) I,\left(a_{4}+b_{4}\right) F\right)$

$a-b=\left(a_{1}-b_{1},\left(a_{2}-b_{2}\right) T,\left(a_{3}-b_{3}\right) I,\left(a_{4}-b_{4}\right) F\right)$.
Definition 4 Let $a=\left(a_{1}, a_{2} T, a_{3} I, a_{4} F\right) \in N Q(X)$ and let $\alpha$ be any scalar which may be real or complex, the scalar product $\alpha . a$ is defined by

$\alpha . a=\alpha .\left(a_{1}, a_{2} T, a_{3} I, a_{4} F\right)$

$$
=\left(\alpha a_{1}, \alpha a_{2} T, \alpha a_{3} I, \alpha a_{4} F\right) \text {. }
$$

If $\alpha=0$, then we have $0 . a=(0,0,0,0)$ and for any non-zero scalars $m$ and $n$ and $b=\left(b_{1}, b_{2} T, b_{3} I, b_{4} F\right)$, we have

$$
\begin{aligned}
(m+n) a & =m a+n a, \\
m(a+b) & =m a+m b, \\
m n(a) & =m(n a), \\
-a & =\left(-a_{1},-a_{2} T,-a_{3} I,-a_{4} F\right) .
\end{aligned}
$$

Example 3 From Example 2, we obtain the following:

$$
\begin{aligned}
x+y & =(2+\sqrt{2})-\frac{15}{4} T-7 I-\frac{35}{6} F . \\
x-y & =(2-\sqrt{2})-\frac{9}{4} T+15 I-\frac{25}{6} . \\
2 i z & =(-4+6 i)+(6+8 i) T-8 I-\left(\frac{1}{3}+\frac{2}{5} i\right) F .
\end{aligned}
$$

Multiplication of two neutrosophic quadruple numbers cannot be carried out like multiplication of two real or complex numbers. To multiply two neutrosophic quadruple numbers $a=\left(a_{1}, a_{2} T, a_{3} I, a_{4} F\right), b=\left(b_{1}, b_{2} T, b_{3} I, b_{4} F\right) \in N Q(X)$, the prevalence order of $\{T, I, F\}$ is required. Consider the following prevalence orders:

(i) Suppose in an optimistic way we consider the prevalence order $T>I>F$. Then we have

$$
\begin{aligned}
T I & =I T=\max \{T, I\}=T, \\
T F & =F T=\max \{T, F\}=T, \\
I F & =F I=\max \{I, F\}=I, \\
T T & =T^{2}=T, \\
I I & =I^{2}=I, \\
F F & =F^{2}=F .
\end{aligned}
$$

Then

$$
\begin{aligned}
a \times b= & \left(a_{1}, a_{2} T, a_{3} I, a_{4} F\right) \cdot\left(b_{1}, b_{2} T, b_{3} I, b_{4} F\right) \\
= & \left(a_{1} b_{1},\left(a_{1} b_{2}+a_{2} b_{1}, a_{2} b_{2}+a_{2} b_{3}\right.\right. \\
& \left.+a_{2} b_{4}+a_{3} b_{2}+a_{4} b_{2}\right) T,\left(a_{1} b_{3}+a_{3} b_{1}\right. \\
& \left.\left.+a_{3} b_{3}+a_{3} b_{4}+a_{4} b_{3}\right) I,\left(a_{1} b_{4}+a_{4} b_{1}+a_{4} b_{4}\right) F\right) .
\end{aligned}
$$

(ii) Suppose in a pessimistic way we consider the prevalence order $T \prec I \prec F$. Then we have 


$$
\begin{aligned}
T I & =I T=\max \{T, I\}=I, \\
T F & =F T=\max \{T, F\}=F, \\
I F & =F I=\max \{I, F\}=F, \\
T T & =T^{2}=T, \\
I I & =I^{2}=I, \\
F F & =F^{2}=F .
\end{aligned}
$$

Then

$$
\begin{aligned}
a \times b= & \left(a_{1}, a_{2} T, a_{3} I, a_{4} F\right) \cdot\left(b_{1}, b_{2} T, b_{3} I, b_{4} F\right) \\
= & \left(a_{1} b_{1},\left(a_{1} b_{2}+a_{2} b_{1}+a_{2} b_{2}\right) T,\right. \\
& \left(a_{1} b_{3}+a_{2} b_{3}+a_{3} b_{1}+a_{3} b_{2}+a_{3} b_{3}\right) I, \\
& \left.\left(a_{1} b_{4}+a_{2} b_{4}, a_{3} b_{4}+a_{4} b_{1}+a_{4} b_{2}+a_{4} b_{3}+a_{4} b_{4}\right) F\right) .
\end{aligned}
$$

Example 4 From Example 2, we obtain the following:

(i) For the prevalence order $T>I>F$, we have

$$
x \times y=\left(2 \sqrt{2},(37-3 \sqrt{2}) T,\left(-\frac{43}{3}+4 \sqrt{2}\right) I,\left(\frac{15}{6}-5 \sqrt{2}\right) F\right) .
$$

(ii) For the prevalence order $T \prec I \prec F$, we have

$$
x \times y=\left(2 \sqrt{2},\left(\frac{3}{4}-3 \sqrt{2}\right) T,(-36+4 \sqrt{2}) I,\left(\frac{695}{12}-5 \sqrt{2}\right) F\right) .
$$

Two neutrosophic quadruple numbers $m=\left(a_{1}, b_{1} T, c_{1} I, d_{1} F\right)$ and $n=\left(a_{2}, b_{2} T, c_{2} I, d_{2} F\right)$ cannot be divided as we do for real and complex numbers. Since the literal neutrosophic components $T, I$ and $F$ are not invertible, the inversion of a neutrosophic quadruple number or the division of a neutrosophic quadruple number by another neutrosophic quadruple number must be carried out a systematic way. Suppose we are to evaluate $m / n$. Then we must look for a neutrosophic quadruple number $p=(x, y T, z I, w F)$ equivalent to $m / n$. In this way, we write $m / n=p$. Then

$\frac{\left(a_{1}, b_{1} T, c_{1} I, d_{1} F\right)}{\left(a_{2}, b_{2} T, c_{2} I, d_{2} F\right)}=(x, y T, z I, w F)$

if and only if

$$
\begin{aligned}
& \left(a_{2}, b_{2} T, c_{2} I, d_{2} F\right)(x, y T, z I, w F) \\
& \quad \equiv\left(a_{1}, b_{1} T, c_{1} I, d_{1} F\right) .
\end{aligned}
$$

Assuming the prevalence order $T>I>F$ and from the equality of two neutrosophic quadruple numbers, we obtain from Eq. (1)

$$
\begin{aligned}
& a_{2} x=a_{1} \\
& b_{2} x+\left(a_{2}+b_{2}+c_{2}+d_{2}\right) y+b_{2} z+b_{2} w=b_{1} \\
& c_{2} x+\left(a_{2}+c_{2}+d_{2}\right) z+c_{2} w=c_{1} \\
& d_{2} x+\left(a_{2}+d_{2}\right) w=d_{1}
\end{aligned}
$$

a system of linear equations in unknowns $x, y, z$ and $w$.
By similarly assuming the prevalence order $T<I \prec F$, we obtain from Eq. (1)

$a_{2} x=a_{1}$

$b_{2} x+\left(a_{2}+b_{2}\right) y=b_{1}$

$c_{2} x+c_{2} y+\left(a_{2}+b_{2}+c_{2}\right) z=c_{1}$

$d_{2} x+d_{2} y+d_{2} z+\left(a_{2}+b_{2}+c_{2}+d_{2}\right) w=d_{1}$

a system of linear equations in unknowns $x, y, z$ and $w$.

Example 5 Let $a=(2,-T, I, 2 F)$ and $b=(1,2 T,-I, F)$ be two neutrosophic quadruple numbers in $N Q(\mathbb{R})$.

(i) For the prevalence order $T>I>F$, we obtain

$\frac{(2,-T, I, 2 F)}{(1,2 T,-I, F)}=\left(2,-\frac{11}{3} T, 3 I, 0 F\right)$.

(ii) For the prevalence order $T \prec I \prec F$, we obtain

$\frac{(2,-T, I, 2 F)}{(1,2 T,-I, F)}=\left(2,-\frac{5}{3} T, \frac{2}{3} I, \frac{1}{3} F\right)$.

Theorem 1 Let $a, b, c, d, n \neq 0$. Then:

(i) $\frac{(n a, n b T, n c I, n d F)}{(a, b T, c I, d F)}=n$.
(ii) $\frac{(n a, n b T, n c l, n d F)}{(n, 0 T, 0 I, 0 F)}=(a, b T, c I, d F)$.

Proof Straightforward.

\section{Neutrosophic Quadruple Algebraic Structures, Neutrosophic Quadruple Algebraic Hyper-structures and NeutroQuadrupleAlgebraicStructures}

\subsection{Neutrosophic Quadruple Algebraic Structures and Neutrosophic Quadruple Algebraic Hyper-structures}

Let $N Q(X)$ be a neutrosophic quadruple set and let *: $N Q(X) \times N Q(X) \rightarrow N Q(X)$ be a classical binary operation on $N Q(X)$. The couple $(N Q(X), *)$ is called a neutrosophic quadruple algebraic structure. The structure $(N Q(X), *)$ is named according to the classical laws and axioms satisfied or obeyed by $*$.

If $*: N Q(X) \times N Q(X) \rightarrow \mathbb{P}(N Q(X))$ is the classical hyper operation on $N Q(X)$. Then the couple $(N Q(X), *)$ is called a neutrosophic quadruple hyper-algebraic structure; and the 
hyper-structure $(N Q(X), *)$ is named according to the classical laws and axioms satisfied by $*$.

If $(N Q(X), *)$ and $(N Q(Y), \circ)$ are two neutrosophic quadruple algebraic structures. The mapping $\phi:(N Q(X), *) \rightarrow(N Q(Y), \circ)$ is called a neutrosophic quadruple homomorphism if $\phi$ preserves $*$, $\circ$ and literal neutrosophic components $T, I$ and $F$ that is if

(i) $\phi(x * y)=\phi(x) \circ \phi(y) \quad \forall x, y \in N Q(X)$.

(ii) $\phi(T)=T$.

(iii) $\phi(I)=I$.

(iv) $\phi(F)=F$.

\section{Theorem 2}

(i) $\quad(N Q(\mathbb{Z}),+),(N Q(\mathbb{Q}),+),(N Q(\mathbb{R}),+)$ and $(N Q(\mathbb{C}),+)$ are abelian groups.

(ii) $(N Q(\mathbb{Z}),+, \times), \quad(N Q(\mathbb{Q}),+, \times), \quad(N Q(\mathbb{R}),+, \times) \quad$ and $(N Q(\mathbb{C}),+, \times)$ are commutative rings.

(iii) $(N Q(\mathbb{Z}), \times)$ is a commutative monoid.

(iv) $(N Q(\mathbb{Z}), \times)$ is not a group.

(v) $(N Q(\mathbb{Z}), \div)$ is not a group

Proof See [7].

\subsection{NeutroQuadrupleAlgebraicStructures}

In this section, unless otherwise stated, the optimistic prevalence order $T>I>F$ will be assumed.

Definition 5 Let $N Q(G)$ be a nonempty set and let *: $N Q(G) \times N Q(G) \rightarrow N Q(G)$ be a binary operation on $N Q(G)$. The couple $(N Q(G), *)$ is called a neutrosophic quadruple group if the following conditions hold:

(QG1) $x * y \in G \forall x, y \in N Q(G)$ [closure law].

(QG2) $\quad x *(y * z)=(x * y) * z \forall x, y, z \in G$ [axiom of associativity].

(QG3) There exists $e \in N Q(G)$ such that $x * e=e * x=x$ $\forall x \in N Q(G)$ [axiom of existence of neutral element].

(QG4) There exists $y \in N Q(G)$ such that $x * y=y * x=e$ $\forall x \in N Q(G)$ [axiom of existence of inverse element], where $e$ is the neutral element of $N Q(G)$. If in addition $\forall x, y \in N Q(G)$, we have

(QG5) $\quad x * y=y * x$, then $(N Q(G), *)$ is called a commutative neutrosophic quadruple group.

Definition 6 [NeutroSophication of the law and axioms of the neutrosophic quadruple].
(NQ(G)1) There exist some duplets $(x, y),(u, v)$, $(p, q), \in N Q(G)$ such that $x * y \in G$ (inner-defined with degree of truth $\mathrm{T}$ ) and $[u * v=$ indeterminate (with degree of indeterminacy I) or $p * q \notin N Q(G)$ (outer-defined/falsehood with degree of falsehood F)] [NeutroClosureLaw].

(NQ(G)2) There exist some triplets $(x, y, z),(p, q, r)$, $(u, v, w) \in N Q(G)$ such that $x *(y * z)=(x * y) * z$ (inner-defined with degree of truth $\mathrm{T}$ ) and $[[p *(q * r)]$ or $[(p * q) * r]=$ indeterminate (with degree of indeterminacy I) or $u *(v * w) \neq(u * v) * w$ (outer-defined/falsehood with degree of falsehood F)] [NeutroAxiom of associativity (NeutroAssociativity)].

(NQ(G)3) There exists an element $e \in N Q(G)$ such that $x * e=e * x=x$ (inner-defined with degree of truth $\mathrm{T}$ ) and $[[x * e]$ or $[e * x]=$ indeterminate (with degree of indeterminacy I) or $x * e \neq x \neq e * x$ (outerdefined/falsehood with degree of falsehood F)] for at least one $x \in N Q(G)$ [NeutroAxiom of existence of neutral element (NeutroNeutralElement)].

(NQ(G)4) There exists an element $u \in N Q(G)$ such that $x * u=u * x=e$ (inner-defined with degree of truth $\mathrm{T})$ and $[[x * u]$ or $[u * x)]=$ indeterminate (with degree of indeterminacy I) or $x * u \neq e \neq u * x$ (outer-defined/falsehood with degre of falsehood F)] for at least one $x \in G$ [NeutroAxiom of existence of inverse element (NeutroInverseElement)] where $e$ is a NeutroNeutralElement in $N Q(G)$.

(NQ(G)5) There exist some duplets $(x, y),(u, v),(p, q) \in N Q(G)$ such that $x * y=y * x$ (inner-defined with degree of truth $\mathrm{T}$ ) and $[[u * v]$ or $[v * u]=$ indeterminate (with degree of indeterminacy I) or $p * q \neq q * p$ (outer-defined/falsehood with degree of falsehood F)] [NeutroAxiom of commutativity (NeutroCommutativity)].

Definition 7 A NeutroQuadrupleGroup $N Q(G)$ is an alternative to the neutrosophic quadruple group $Q(G)$ that has at least one NeutroLaw or at least one of $\{N Q(G) 1, N Q(G) 2$, $N Q(G) 3, N Q(G) 4\}$ with no AntiLaw or AntiAxiom.

Definition 8 A NeutroCommutativeQuadrupleGroup $N Q(G)$ is an alternative to the commutative neutrosophic quadruple group $Q(G)$ that has at least one NeutroLaw or at least one of $\{N Q(G) 1, N Q(G) 2, N Q(G) 3, N Q(G) 4\}$ and $N Q(G) 5$ with no AntiLaw or AntiAxiom.

Theorem 3 [15] Let $\mathbb{U}$ be a nonempty finite or infinite universe of discourse and let $S$ be a finite or infinite subset of $\mathbb{U}$. If $n$ classical operations (laws and axioms) are defined on $S$ where $n \geq 1$, then there will be $\left(2^{n}-1\right)$ NeutroAlgebras and $\left(3^{n}-2^{n}\right)$ AntiAlgebras. 
Theorem 4 Let $(N Q(G), *)$ be a neutrosophic quadruple group. Then

(i) there are 15 types of NeutroQuadrupleGroups,

(ii) there are 31 types of NeutroCommutativeQuadrupleGroups.

Proof Follows from Theorem 3.

Theorem 5 For positive integers $n=2,3,4, \cdots$,

(i) $\left(N Q\left(\mathbb{Z}_{n}\right),-\right)$ is a NeutroQuadrupleGroup.

(ii) $\left(N Q\left(\mathbb{Z}_{n}\right), \times\right)$ is a NeutroCommutativeQuadrupleGroup.

Proof Follows from the definition of NeutroQuadrupleGroup and subtraction and multiplication of neutrosophic quadruple of integers modulo $n$.

\section{Theorem 6}

(i) $(N Q(\mathbb{Z}),-)$ is a NeutroQuadrupleGroup.

(ii) $(N Q(\mathbb{Z}), \times)$ is a NeutroCommutativeQuadrupleGroup.

(iii) $(N Q(\mathbb{Z}), \div)$ is a NeutroCommutativeQuadrupleGroup.

Proof (i) and (ii) are easy. For (iii), let us consider the following:

\section{NeutroClosure of $\div$ over $N Q(\mathbb{Z})$}

For the degree of truth, let $a=(0,0 T, I, 0 F) \in N Q(\mathbb{Z})$. Then $a \div a=\left(1-k_{1}-k_{2}, 0 T, k_{1} I, k_{2} F\right) \in N Q(\mathbb{Z}), k_{1}, k_{2} \in \mathbb{Z}$. For the degree of indeterminacy, let $a=(4,5 T,-2 I,-7 F), b=(0,-6 T, I, 3 F) \in N Q(\mathbb{Z})$. Then $a \div b=\left(\frac{4}{0}, ? T, ? I, ? F\right) \notin N Q(\mathbb{Z})$.

For the degree of falsehood, let $a=(0,0 T, 0 I, F)$, $b=(0,0 T, 0 I, 2 F) \in N Q(\mathbb{Z})$. Then

$$
a \div b=\left(\frac{1}{2}-k, 0 T, 0 I, k F\right) \notin N Q(\mathbb{Z}), k \in \mathbb{Z} \text {. }
$$

\section{NeutroAssociativity of $\div$ over $N Q(\mathbb{Z})$}

For the degree of truth, let $a=(6,6 T, 6 I, 6 F)$, $b=(2,2 T, 2 I, 2 F), c=(-1,0 T, 0 I, 0 F) \in N Q(\mathbb{Z})$. Then

$a \div(b \div c)=(-3,0 T, 0 I, 0 F)$, b u $\quad \mathrm{t}$

$(a \div b) \div c=(-3,0 T, 0 I, 0 F)$.

For the degree of indeterminacy, let

$$
a=(4,-T, 2 I,-7 F), \quad b=(0, T, 0 I,-8 F),
$$

$c=(0,0 T, 9 I,-F) \in N Q(\mathbb{Z})$. Then

$$
a \div(b \div c)=(?, ? T, ? I, ? F) \text {. }
$$

$(a \div b) \div c=(?, ? T, ? I, ? F)$.
For the degree of falsehood, let $a=(0,5 T, 0 I, 0 F)$, $b=(0, T, 0 I, 0 F), c=(5,0 T, 0 I, 0 F) \in N Q(\mathbb{Z})$. Then

$a \div(b \div c)=\left(25-k_{1}-k_{2}-k_{3}, k_{1} T, k_{2} I, k_{3} F\right) \in N Q(\mathbb{Z}), k_{1}, k_{2}, k_{3} \in \mathbb{Z}$. $(a \div b) \div c=\left(\frac{1}{5}\left(5-k_{1}-k_{2}-k_{3}\right), \frac{1}{5} k_{1} T, \frac{1}{5} k_{2} I, \frac{1}{5} k_{3} F\right) \notin N Q(\mathbb{Z})$.

Existence of NeutroUnitaryElement and NeutroInverseElement in $N Q(\mathbb{Z})$ w.r.t. $\div$

L e t $\quad a=(0, T, 0 I, 0 F), \quad b=(0,0 T, I, 0 F)$, $c=(0,0 T, 0 I, F) \in N Q(\mathbb{Z})$. Then

$a \div a=\left(1-k_{1}-k_{2}-k_{3}, k_{1} T, k_{2} I, k_{3} F\right)$

$b \div b=\left(1-k_{1}-k_{2}, 0 T, k_{1} I, k_{2} F\right)$

$c \div c=(1-k, 0 T, 0 I, k F)$

$a \div b=\left(-\left(k_{1}+k_{2}\right), T, k_{1} I, k_{2} F\right)$

$b \div a=\left(-\left(k_{1}+k_{2}+k_{3}\right), k_{1} T, k_{2} I, k_{3} F\right)$

where $k, k_{1}, k_{2}, k_{3} \in \mathbb{Z}$.

For the degree of truth, putting $k_{1}=1, k_{2}=k_{3}=0$ in Eq. (1), $k_{1}=1, k_{2}=0$ in Eq. (2) and $k=1$ in Eq. (3) we will obtain $a \div a=a, b \div b=b$ and $c \div c=c$. These show that $a, b, c$ are, respectively, NeutroUnitaryElements and NeutroInverseElements in $N Q(\mathbb{Z})$.

For the degree of falsehood, putting $k_{1} \neq 1, k_{2} \neq k_{3} \neq 0$ in Eq. (1), $k_{1} \neq 1, k_{2} \neq 0$ in Eq. (2) and $k \neq 1$ in Eq. (3) we will obtain $a \div a \neq a, b \div b \neq b$ and $c \div c \neq c$. These show that $a, b, c$ are, respectively, not NeutroUnitaryElements and NeutroInverseElements in $N Q(\mathbb{Z})$.

\section{NeutroCommtativity of $\div \operatorname{over} N Q(\mathbb{Z})$}

For the degree of truth, putting $k_{1}=1, k_{2}=k_{3}=0$ in Eq. (1), $k_{1}=1, k_{2}=0$ in Eq. (2) and $k=1$ in Eq. (3) we will obtain $a \div a=a, b \div b=b$ and $c \div c=c$. These show the commutativity of $\div$ wrt $a, b$ and $c N Q(\mathbb{Z})$.

For the degree of falsehood, putting $k_{1}=k_{2}=k_{3}=1$ in Eqs. (4) and (5), we will obtain $a \div b=(-2, T, I, F)$ and $b \div a=(-3, T, I, F) \neq a \div b$. Hence, $\div$ is NeutroCommutative in $N Q(\mathbb{Z})$.

The proof is complete.

Definition 9 Let $(N Q(G), *)$ be a neutrosophic quadruple group. A nonempty subset $N Q(H)$ of $N Q(G)$ is called a NeutroQuadrupleSubgroup of $N Q(G)$ if $(N Q(H), *)$ is a neutrosophic quadruple group of the same type as $(N Q(G), *)$.

\section{Example 6}

(i) For $n=2,3,4, \cdots(N Q(n \mathbb{Z}),-)$ is a NeutroQuadrupleSubgroup of $(N Q(\mathbb{Z}),-)$. 
(ii) For $n=2,3,4, \cdots(N Q(n \mathbb{Z}), \times)$ is a NeutroQuadrupleSubgroup of $(N Q(\mathbb{Z}), \times)$.

\section{Example 7}

(i) Let $N Q(H)=\{(a, b T, c I, d F): a, b, c, d \in\{1,2,3\}\}$ be a subset of the NeutroQuadrupleGroup $\left(N Q\left(\mathbb{Z}_{4}\right),-\right)$. Then $(N Q(H),-)$ is a NeutroQuadrupleSubgroup of $\left(N Q\left(\mathbb{Z}_{4}\right),-\right)$.

(ii) Let $N Q(K)=\{(w, x T, y I, z F): a, b, c, d \in\{1,3,5\}\}$ be a subset of the NeutroQuadrupleGroup $\left(N Q\left(\mathbb{Z}_{6}\right), \times\right)$. Then $(N Q(H), \times)$ is a NeutroQuadrupleSubgroup of $\left(N Q\left(\mathbb{Z}_{6}\right), \times\right)$.

\section{Conclusion}

We have in this paper studied neutrosophic quadruple algebraicstructures and NeutroQuadrupleAlgebraicStructures. NeutroQuadrupleGroup was studied in particular and several examples were provided. It was shown that $(N Q(\mathbb{Z}), \div)$ is a NeutroQuadrupleGroup. Substructures of NeutroQuadrupleGroups were also presented with examples.

Author Contributions FS, AR, AAAA, YBJ, RAB, BD, ABS, MA, $\mathrm{MH}, \mathrm{SM}$ contributed equally to this work.

\section{Declarations}

Conflict of interest The authors declare that they have no conflict of interest.

Open Access This article is licensed under a Creative Commons Attribution 4.0 International License, which permits use, sharing, adaptation, distribution and reproduction in any medium or format, as long as you give appropriate credit to the original author(s) and the source, provide a link to the Creative Commons licence, and indicate if changes were made. The images or other third party material in this article are included in the article's Creative Commons licence, unless indicated otherwise in a credit line to the material. If material is not included in the article's Creative Commons licence and your intended use is not permitted by statutory regulation or exceeds the permitted use, you will need to obtain permission directly from the copyright holder. To view a copy of this licence, visit http://creativecommons.org/licenses/by/4.0/.
2. Agboola, A.A.A.: Introduction to NeutroGroups. Int. J. Neutrosophic Sci. f6(1), 41-47 (2020)

3. Agboola, A.A.A.: On finite neutrogroups of type-NG[1,2,4]. Int. J. Neutrosophic Sci. 10(2), 84-95 (2020)

4. Agboola, A.A.A.: Introduction to NeutroRings. Int. J. Neutrosophic Sci. 7(2), 62-73 (2020)

5. Agboola, A.A.A., Ibrahim, M.A.: Introduction to AntiRings. Neutrosophic Sets Syst. 36, 293-307 (2020)

6. Agboola, A.A.A., Davaz, B., Smarandache, F.: Neutrosophic quadruple algebraic hyperstructures. Ann. Fuzzy Math. Inf. 14, 29-42 (2017)

7. Akinleye, S.A., Smarandache, F., Agboola, A.A.A.: On neutrosophic quadruple algebraic structures. Neutrosophic Sets Syst. 12, 122-136 (2016)

8. Akram, M., Shum, K.P.: A survey on single-valued neutrosophic K-algebras. J. Math. Res. Appl. 40(3) (2020)

9. Akram, M., Gulzar, H., Smarandache, F., Broumi, S.: Certain notions of neutrosophic topological K-algebras. Mathematics 6(11), 234 (2018)

10. Akram, M., Gulzar, H., Smarandache, F., Broumi, S.: Application of neutrosophic soft sets to K-Algebras. Axioms 7(4), 83 (2018)

11. Al-Tahan, M., Davvaz, B.: On some properties of neutrosophic quadruple Hv-rings. Neutrosophic Sets Syst. 36, 256-270 (2020)

12. Borzooei, R.A., Mohseni Takallo, M., Smarandache, F., Jun, Y.B.: Positive implicative BMBJ-neutrosophic ideals in BCK-algebras. Neutrosophic Sets Syst. 23, 126-141 (2018)

13. Hamidi, M., Smarandache, F.: Neutro-BCK-algebra. Int. J. Neutrosophic Sci. 8, 110-117 (2020)

14. Jun, Y.B., Song, S.Z., Smarandache, F., Bordbar, H.: Neutrosophic quadruple BCK/BCI-algebras. Axioms 7(41) (2018)

15. Rezaei, A., Smarandache, F.: On neutro-BE-algebras and anti-BEalgebras. Int. J. Neutrosophic Sci. 4, 8-15 (2020)

16. Smarandache, F.: NeutroAlgebra is a generalization of partial algebra. Int. J. Neutrosophic Sci. 2, 8-17 (2020)

17. Smarandache, F.: Generalizations and alternatives of classical algebraic structures to neutroalgebraic structures and antialgebraic structures. J. Fuzzy Ext. Appl. 1(2), 85-87 (2020)

18. Smarandache, F.: Introduction to NeutroAlgebraic Structures and AntiAlgebraic Structures. In: Advances of Standard and Nonstandard Neutrosophic Theories, Chapter 6, pp. 240-265. Pons Publishing House, Brussels (2019)

19. Smarandache, F.: Neutrosophic quadruple numbers, refined neutrosophic quadruple numbers, absorbance law, and the multiplication of neutrosophic quadruple numbers, neutrosophic sets and systems, vol. 10, pp. 96-98 (2015)

20. Smarandache, F., Rezaei, A., Kim, H.S.: A new trend to extensions of CI-algebras. Int. J. Neutrosophic Sci. 5(1), 8-15 (2020)

21. UNM: Paradoxism: the last vanguard of second millennium (2021). http://fs.unm.edu/a/paradoxism.htm

Publisher's Note Springer Nature remains neutral with regard tojurisdictional claims in published maps and institutional affiliations.

\section{References}

1. Agboola, A.A.A., Ibrahim, M.A., Adeleke, E.O.: Elementary examination of neutroalgebras and antialgebras viz-a-viz the classical number systems. Int. J. Neutrosophic Sci. 4(1), 16-19 (2020) 